\title{
Inhibition of action rules
}

\author{
ULRICH MAYR \\ University of Oregon, Eugene, Oregon
}

\begin{abstract}
Inhibition is often proposed as an important executive-control process, but its existence is difficult to establish empirically. Recently, Mayr and Keele (2000)reported that it takes longer to switch to a recently disengaged task set (i.e., $n-2$ task-set repetitions) than to a less recently disengaged task set. This setalternation cost may indicate inhibition of the disengaged task set. The primary goal of the present study was to test the inhibition account of set-alternation costs against an important alternativeaccount based on automatic episodic retrieval. The episodic retrievalaccount predicts reaction time facilitation instead of costs for the special case of complete $n-2$ repetitions of both task sets and all stimulusresponse aspects. A new task-switching paradigm, in which action rules varied on a trial-by-trial basis while the relevant stimulus dimension remained invariant, allowed the implementation of a high proportion of complete $n-2$ repetitions. Consistent with the inhibition view, set-alternation costs were obtained even for these constellations.
\end{abstract}

The space of potential stimuli to attend to and actions to perform is infinite. Therefore, coherent, goal-directed action is conditional on internal constraints on perception and action, often referred to as mental sets or task sets (e.g., Logan, 1978; Woodworth, 1938). What are the processes associated with selection of task sets? This question has recently become a major topic of investigation in the context of research with the so-called task-switching paradigm (Allport, Styles, \& Hsieh, 1994; Mayr \& Kliegl, 2000; Meiran, 1996; Rogers \& Monsell, 1995). At the heart of the task-switching paradigm is an experimental contrast between trial-to-trial transitions in which the task set is either invariant or needs to be changed. The reaction time (RT) and performance costs that arise at switch points (switch costs) are thought to reflect processes involved in the selection of task sets.

One particular set-selection problem has been referred to as the stability-flexibility dilemma (e.g., Goschke, 2000; Mayr \& Keele, 2000). On the one hand, task sets need to be highly activated to allow reliable guidance of lowlevel selection in the face of ambiguous and fluctuating environmental stimulation. On the other hand, when changes in the course of action become necessary, highly activated task sets may stand in the way of behavioral flexibility. A solution to the dilemma could be an inhibitory process that actively suppresses the last relevant task set, thereby clearing the slate for the establishment of the next set.

Mayr and Keele (2000) have recently proposed a paradigm that provides a test of this set-inhibitionidea. Specifically, inhibition of task sets is inferred from the finding that switching back to a task set that has been abandoned two

This research was funded through the Deutsche Forschungsgemeinschaft (Grant INK 12/A1, Project C). I thank Petra Grüttner for running the experiments and Steve Keele for valuable comments. Correspondence should be addressed to U. Mayr, Department of Psychology, University of Oregon, Eugene, OR 97403 (e-mail: mayr@oregon.uoregon.edu). trials earlier (i.e., an A-B- $A$ sequence of task sets) takes more time than switching back to a task set that has occurred less recently (i.e., a $\mathrm{C}-\mathrm{B}-A$ sequence of task sets). The interpretation of this set-alternation cost is that during disengagement, a task set receives an inhibitory input that makes reactivation of that task set more difficult for some period of time.

Whether or not inhibitionis claimed to be a means of selection between competing codes marks a dividing line between broad classes of theoretical models of cognitive control. Models rooted in the spreading-activation and symbol-processing tradition often explicitly avoid inhibition as a selection device (e.g., J. R. Anderson, 1993; Just \& Carpenter, 1992; Kimberg \& Farah, 1993). In contrast, inhibition is an intricate part of models that rely on constraint satisfaction between mutually associated codes (e.g., Houghton \& Tipper, 1994) or of models that claim neurobiological plausibility (e.g., Amos, 2000; Dehaene, Kerszeberg, \& Changeux, 1998). Therefore, it is a theoretically important question whether the set-alternation cost actually does reflect "true inhibition" or whether alternative, noninhibitory processes can be held responsible.

One process that, at least in theory, can produce inhibitionlike phenomena is the stimulus-triggered retrieval of earlier action episodes associated with that stimulus (Neill, 1997). This so-called episodic-retrieval account has been applied with at least partial success to negative priming, one of the most vigorously studied inhibitory phenomena (see, e.g., Tipper \& Cranston, 1985). Negative priming denotes the slowing of response times to a target stimulus on trial $n$ that was a distractor on trial $n-1$ and which therefore may still be in an inhibited state. According to the episodic-retrieval account of this result, an episodic-memory record is created during trial $n-1$ that contains the distractor stimulus as well as the associated action-namely, not to respond to that stimulus. If the same stimulus becomes a target on trial $n$, this "do-not-respond" tag is re- 
trieved automatically and interferes with the adequate response.

A variant of the episodic-retrieval account could also explain the set-alternation cost. From an episodic-retrieval view, processing in a set-alteration sequence $(\mathrm{A}-\mathrm{B}-\mathrm{A})$ might produce a mismatch cost relative to the processing in a no-alternation sequence $(\mathrm{C}-\mathrm{B}-\mathrm{A})$ if critical parameters, such as the response to be made under set $\mathrm{A}$, differ on the two alternation trials. Note also that from an episodicretrieval view, processing in a set-alternation sequence should benefit relative to the no-alteration control if the response is the same on the two alternation trials. In the experiments reported by Mayr and Keele (2000), stimuli were randomly determined within a multidimensional stimulus space. As a result, stimulus-response aspects almost never repeated completely across $n-2$ repetitions of the same task set. Thus, at least in principle, the findings reported by Mayr and Keele are open to interpretation from an episodic retrieval perspective.

\section{Is the Set-Alternation Cost Due to Episodic Retrieval?}

The empirical distinction between the inhibition account and the episodic retrieval account has proven very difficult for the negative priming effect (Kane, May, Hasher, \& Rahal, 1997; Neill, 1997). In contrast, an empirical decision between these two views seems relatively straightforward in the context of a new rule-selection paradigm that is used in the present study. Illustrative sequences of trials from the rule-selection task are shown in Figure 1. Task sets were three different simple stimulus-response rules (horizontal, vertical, or diagonal spatial translations). Each trial began with the presentation of the instructional cue for the to-beused rule (in form of a verbal label) and then of the stimulus, a circle presented in one of the corners of a square. Responses had to be entered on one of four keys arranged in terms of a $2 \times 2$ matrix, conditional on the circle position in one of four corners of a square and on the currently relevant stimulus-response rule provided by the instructional cue. Note, that there are three components to each trial: the rule, the stimulus (the location of the circle), and the response (which key defines the correct response). From an episodic-retrieval view, processing in a set-alternation sequence of rules (e.g., horizontal-vertical-horizontal) could be described in the following manner: During processing of the first occurrence of the horizontal rule, relevant lower-level aspects, such as the actual stimulus and the actual response are encoded. Two trials later, when the same rule needs to be applied again, these aspects would be automatically retrieved. If the retrieved and the actually required stimulus-response parameters do not match (i.e., the $n-2$ stimulus-response change case in Figure 1), a mismatch cost could arise. Note that in the control condition, in which there is no $n-2$ repetition on the level of sets, one would not expect an equivalent mismatch cost if one assumes that stimulus-response aspects actually become associated with the relevant task set.
How can the episodic-retrieval explanation be distinguished from the inhibition account? The key is the examination of stimulus-response similarity across repeated sets. If a set-alternation cost were to be found only for $n-2$ set repetitions that go along with mismatching stimuli, but a positive priming effect for set repetitions that go along with matching stimuli, this would point to the contribution of set-specific episodic retrieval. However, if a set-alternation cost were found throughout, true inhibition would seem to be much more likely. The strongest possible evidence would of course be the finding of a set-alternation cost even in the case of complete repetitions of all $n-2$ stimulus aspects (i.e., the $n-2$ stimulus-response and rule repetition case in Figure 1). In the rule-selection paradigm, only four different stimuli are used (i.e., positions of

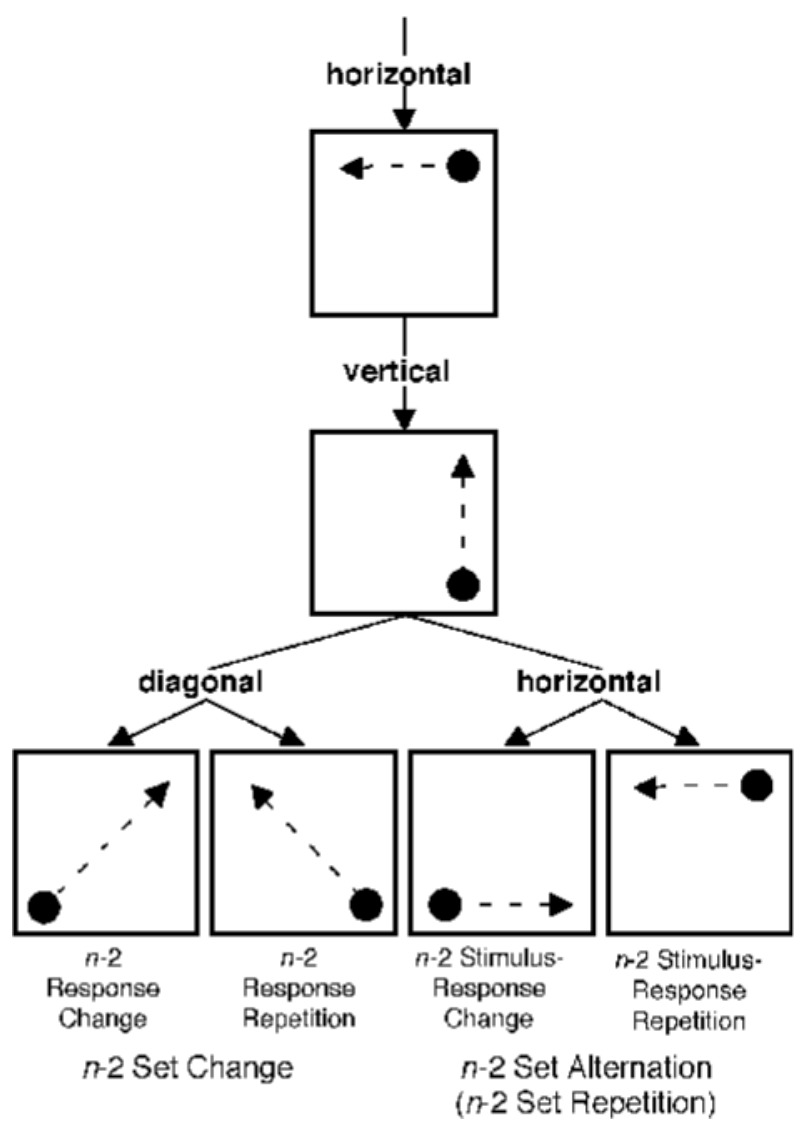

Figure 1. Sample switch trials in which, on trial $n$, either there was no set-alternation (i.e., the rules on trial $n-2$ and $n$ were different) or there was a set alternation (i.e., the rule on trial $n-2$ was repeated on trial $n$ ), with and without repetition of the trial $n-2$ response on trial $n$. The episodic-retrieval account predicts fastest reaction times (RTs) for the $n-2$ set alternation with $n-2$ response repetition. In contrast, the inhibition account predicts slower RTs with the $n-2$ set alternation both with and without response repetition. The dashed arrows within stimulus frames illustrate the correct response key, but were not part of the stimulus. Note that the four response keys were arranged compatibly with potential circle positions. 
the circle within the frame), so that complete repetitions of $n-2$ stimulus aspects occur with a relatively high probability of $p=.25$. Thus, the critical question is whether or not, for the $n-2$ stimulus-response-repetition case, setalternation costs or set-alternation benefits are obtained.

\section{Inhibition of Task Sets or Perceptual Modules?}

The rule-selection paradigm also addresses another open issue concerning the generality of the set-alternation cost. Mayr and Keele (2000) interpreted the set-alternation cost in terms of inhibition applied to the representation of abstract task sets or rules (see also, Amos, 2000). However, the nature of the tasks used by Mayr and Keele leaves an alternative interpretation. Specifically, participants had to select on a trial-by-trial basis task sets that specified the relevant perceptual dimension (i.e., color, movement, and orientation) on which a deviant object had to be detected and located. Thus, the task sets were actually perceptual sets, and every switch in set involved a switch between perceptual dimensions. Further, each of the perceptual dimensions were associated with distinct neuroanatomic processing sites, leaving the possibility that set-level inhibition may be operating through the top-down modulation of low-level, dimension-specific processing sites (Corbetta, Miezin, Dobmeyer, Shulman, \& Petersen, 1991). Importantly, in the rule-selection paradigm, the task sets are simple spatial response rules, so that task-set switches are not confounded with switches in perceptual dimensions. For each rule alike, it is only the position of the circle within the frame that is relevant so that top-down modulations of dimension-specific processing sites cannot be a critical aspect of set switching here. Thus, the finding of set-alternation costs in this situation would lend strong support to the claim that inhibition can affect setlevel representations.

Finally, given that this is the first use of the rule-selection paradigm, it seemed important to examine whether it allows replication of typical task-switching effects. One of the most important variables in this context is the time provided for preparing for the next trial, operationalized as the interval between task cue and imperative stimulus (or the cue-stimulus interval, CSI). Specifically, if processes associated with switch costs and set-alternation costs are under intentional control, they should be affected by the CSI. Past work has revealed that switch costs are at least partially reduced as the CSI increases (e.g., Mayr \& Kliegl, 2000; Meiran, 1996; Rogers \& Monsell, 1995), whereas the set-alternation cost seemed unaffected by this variable (Mayr \& Keele, 2000). Set-alternation costs have, however, been found to be sensitive to the total intertrial interval, no matter whether it could be used for active preparation or not (Mayr \& Keele, 2000). To assess the effects of active preparation and passive decay, both the interval between the response to the preceding trial and the task cue (the response-cue interval, RCI) and the interval between the task cue and the stimulus (CSI) were manipulated in the present experiment (for the use of similar designs, see Mayr \& Kliegl, 2000, and Meiran, 1996).

\section{METHOD}

\section{Participants}

Thirty-nine students at the University of Potsdam participated in a single-session experiment in exchange for course credit or DM 10 $(\sim \$ 6)$.

\section{Task and Stimuli}

Stimulus presentation occurred on a 17 -in. Macintosh monitor. The stimulus display contained a frame in the form of a square with a side length of $8 \mathrm{~cm}$, which with a viewing distance of about $50 \mathrm{~cm}$, subtended $9.1^{\circ}$. On each trial, a circle with a diameter of $1 \mathrm{~cm}$ appeared in one of the four corners of the frame (see Figure 1). Responses were entered on four keys on the standard Macintosh keyboard with the same spatial arrangement as the four object locations ("1," " 2 ," " 4 ," and " 5 "). The participants were instructed to rest their index fingers of their preferred hand in the middle between the four keys and to move their fingers to the correct key. After pressing the key, the participants were supposed to bring back their fingers to their center positions. Correct keys were cospecified by the object location and the currently relevant action rule. In the case of the horizontal rule, the response location could be derived by translating the circle position horizontally (i.e., the upper right key in the case of an upper left circle position). In the case of the vertical rule, the response location was vertically translated relative to the circle position (i.e., the lower left key in the case of an upper left circle position), and in the case of the diagonal rule, the response location was diagonally translated relative to the circle position (i.e., the lower right key in the case of an upper left circle position). A single word that indicated the action rule (horizontal, vertical, or diagonal) for the current item was presented immediately above the stimulus frame. When there was an incorrect response, the word "Error" was presented on the screen for $500 \mathrm{msec}$.

Relevant action rules and stimulus locations were selected randomly for each trial without constraints. The time between a response and the cue for the next display could be either 150 or $650 \mathrm{msec}$ (RCI). Also, the time between the cue and the next stimulus display could be either 150 or $650 \mathrm{msec}$ (CSI). Of the four possible RCI-CSI combinations, three were implemented: 150-150, $650-150$, and $150-650 .^{1}$

\section{Procedure}

At the beginning of the session, the participants were familiarized with the paradigm in at least two 10-trial practice blocks, which could be repeated on demand. Instructions emphasized both speed and accuracy. Actual testing occurred in three sets of three blocks of 120 trials per block. For each set of three blocks, a fixed RCI-CSI constellation was used. In order to become familiarized with the RCI-CSI constellation of the following block, the participants went through 10 practice trials prior to each block. The sequence of RCI-CSI constellation cycles was counterbalanced across participants.

\section{RESULTS AND DISCUSSION}

Error trials and trials after an error within the preceding two trials were eliminated (i.e., $8.4 \%$ of all trials), as well as all RTs larger than $2,000 \mathrm{msec}$ (i.e., $0.3 \%$ of all trials). Overall error rate was low $(M=3.2 \%, S D=2.1)$, and unless reported otherwise, the error pattern was in the same direction as the RT effects (error data are presented in Figure 2).

\section{Switch Costs}

It seemed important to examine in an initial set of analyses to what degree typical switch-cost effects could 


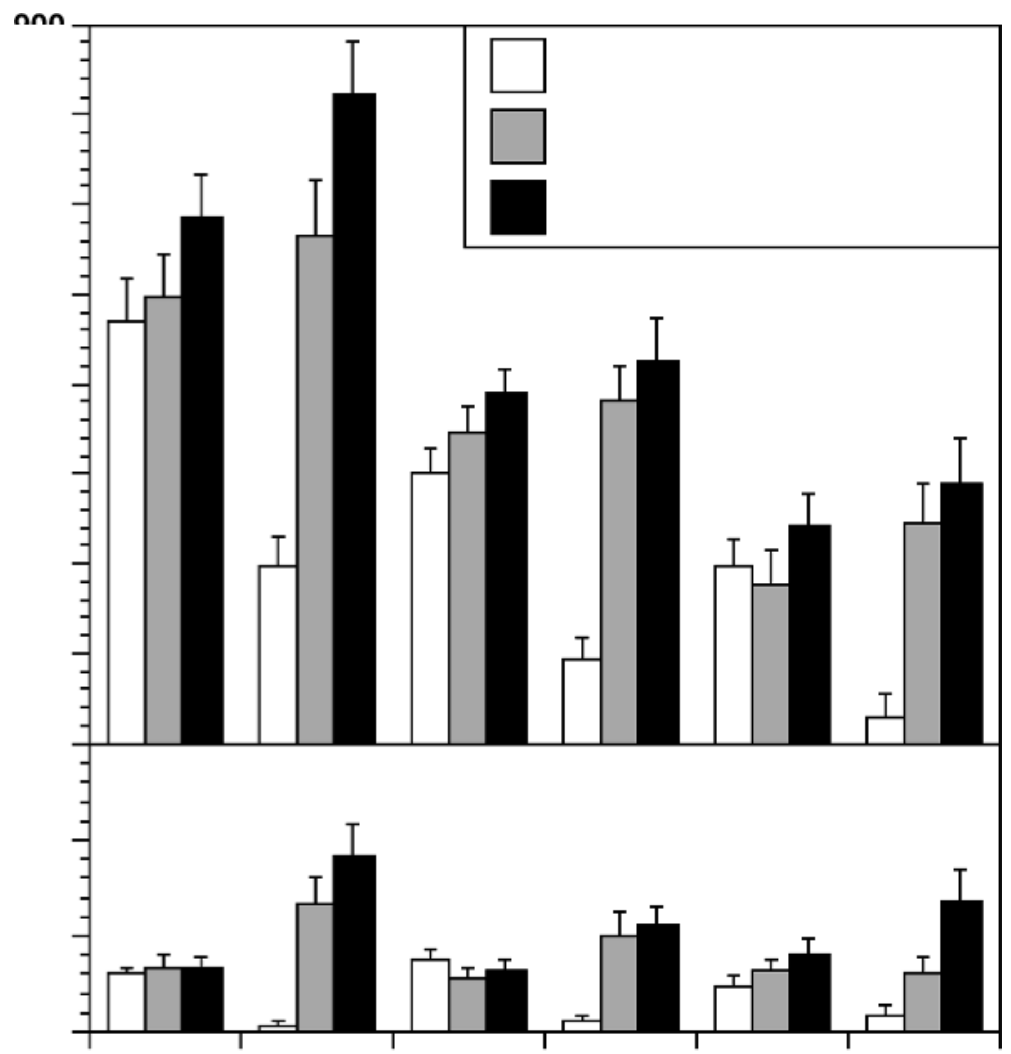

Figure 2. Reaction times and error percentages for no-switch trials, switch trials without set alternations, and switch trials with set alternations as a function of RCI-CSI constellations and the response-repetition variable. Error bars reflect between-subjects standard errors.

be replicated in the rule-selection paradigm. Each single trial was classified dependent on the task sets (i.e., rules) in the preceding two trials into three categories: (1) same rule as in the immediately preceding trial (e.g., no-switch: diagonal-vertical-vertical), (2) switch to the rule not used in the preceding two trials (e.g., nonalternating switch: diagonal-vertical-horizontal), and (3) switch back to the rule used two trials earlier (e.g., alternating switch: diagonal-vertical-diagonal). Note, that the set-alternation effect can be analyzed only for trial triplets, with different rules on triplet Positions 1 and 2. In order to achieve comparability across all three trial categories, we omitted all trials that followed a rule repetition (e.g., verticalvertical-vertical or vertical-vertical-diagonal) from further analysis.

As an additional classification criterion, immediate (i.e., $n-1)$ response-repetition trials were distinguished from response-change trials (not to be confused with the $n-2$ response-repetition variable to be examined below). The reason for including this factor was that immediate response repetitions have been found to produce strong interactions with the switch contrast (e. g., Meiran, 1996; Quinlan, 1999; Rogers \& Monsell, 1995). Specifically, typical response-priming effects are absent or even reversed in case of a task-set switch. Therefore it seemed critical to examine in which way theoretically important effects are modulated by this factor.

Figure 2 shows RTs for all critical design cells. These results were analyzed using two sets of two nonorthogonal contrasts along with the response-repetition variable. The first set compared (1) no-switch and nonalternating switch trials (i.e., switch contrast) and (2) nonalternating and alternating switch trials (i.e., set-alternation contrast). The second set compared (1) the 150-150 and the 650-150 RCI-CSI constellations (i.e., decay contrast) and (2) the 650-150 and the 150-650 RCI-CSI constellations (i.e., preparation contrast). The switch and the set-alternation contrasts tested the overall switch and set-alternation effects; their interactions with decay and preparation contrast as well as with the response-repetition variable tested the degree to which switch costs and set-alternation costs are influenced by passive decay, active preparation, and response repetitions.

Changes in task sets led to highly significant switch costs [switch contrast: $F(1,38)=54.9, M S_{\mathrm{e}}=14,5108.0$, $p<.01]$, which were almost significantly reduced as a 
function of decay interval [switch contrast $\times$ decay contrast: $\left.F(1,38)=3.8, M S_{\mathrm{e}}=16,627.1, p<.06\right]$ and were significantly reduced as a function of preparatory interval [switch contrast $\times$ preparation contrast: $F(1,38)=9.1$, $\left.M S_{\mathrm{e}}=14,4330.4, p<.01\right]$. The latter result generalizes the findings by Meiran (1996) and Rogers and Monsell (1995) of an endogenous set-configuration process to the present situation, in which simple action rules rather than complete task sets had to be changed. It should be also noted that both the RCI and the CSI had strong effects on overall RTs [decay contrast: $F(1,38)=29.7, M S_{\mathrm{e}}=$ $430,554.3, p<.01$; preparation contrast: $F(1,38)=19.3$, $\left.M S_{\mathrm{e}}=259,851.4, p<.01\right]$.

In replication of other task-switching studies, switch costs were modulated by response repetitions (e.g., Meiran, 1996; Quinlan, 1999; Rogers \& Monsell, 1995) [switch contrast $\times$ response-repetition variable: $F(1,38)=86.9$, $\left.M S_{\mathrm{e}}=78,652.9, p<.01\right]$. As is evident from Figure 2, switch costs (i.e., the difference between no-switch and no-alternation switches) were very small for response changes both in the $150-150$ constellation $(13 \mathrm{msec})$ and in the $650-150$ constellation $(13 \mathrm{msec})$ and they actually became negative for the condition with the long preparatory interval $(-9 \mathrm{msec})$, a finding that is, however, offset by a reverse trend for errors. In contrast, for response repetitions, switch costs were generally large (184 and $143 \mathrm{msec}$ for the 150-150 and the 650-150 constellations, respectively), and even with preparation, there was a residual cost of 108 msec. Inspection of Figure 2 suggests that the relatively large switch costs in case of response repetitions can be attributed mostly to facilitation in the case of no- switch transitions rather than to costs in the case of switch transitions.

The small switch costs obtained in this experiment, in particular in the absence of response repetitions, may seem surprising at first. However, this result is consistent with an account of switch costs in terms of long-term memory retrieval demands (Mayr \& Kliegl, 2000; Rubinstein, Meyer, \& Evans, 2001). In typical switching paradigms, not only the relevant stimulus dimension but also arbitrary stimulus-response (S-R) mappings have to be retrieved (e.g., Rogers \& Monsell, 1995). In contrast, in the present paradigm, retrieval demands were small because only a very simple response rule had to be retrieved on the basis of a direct verbal cue.

\section{Set-Alternation Costs}

As is evident from Figure 2, RTs for set-alternation switches were larger than for nonalternating switches in all conditions [set-alternation contrast: $F(1,38)=31.6, M S_{\mathrm{e}}=$ $61,115.1, p<.01]$. The set-alternation cost became significantly smaller for longer decay intervals [set-alternation contrast $\times$ decay contrast: $F(1,38)=6.4, M S_{\mathrm{e}}=36,865.6$, $p<.05$ ], a finding that replicates results reported by Mayr and Keele (2000; Experiment 1b). In contrast, the setalternation cost was not affected by opportunity for preparation [set-alternation contrast $\times$ preparation contrast: $\left.F(1,38)=0.1, M S_{\mathrm{e}}=22,299.9, p>.7\right]$. This result, which also replicates the findings by Mayr and Keele (2000), is important because it indicates that the potential inhibitory process cannot be easily overruled by active preparation (for more extensive discussion of this aspect, see Mayr \&

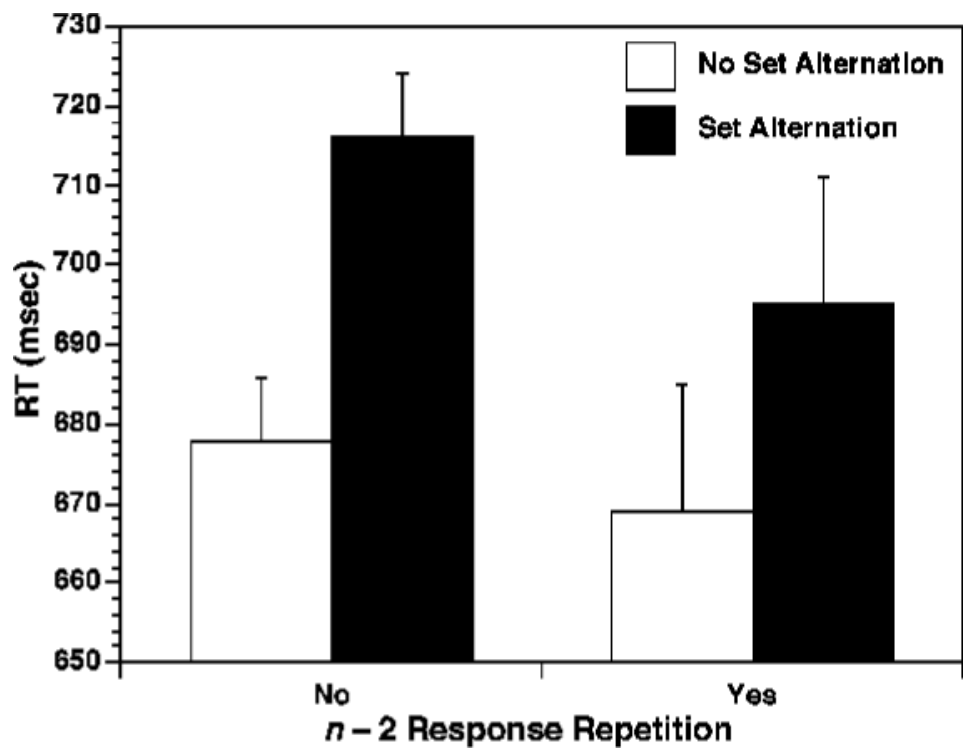

Figure 3. Reaction times from switch trials without set alternations $(n-2$ rule changes) and with set alternations ( $n-2$ rule repetitions) as a function of $n-2$ response repetitions. Error bars reflect within-subjects $95 \%$ confidence intervals for the set-alternation contrast, computed separately for $n-2$ response changes and for $n-2$ response repetitions. 
Keele, 2000). It also rules out an interpretation of the setalternation cost in terms of sequential expectancies. ${ }^{2}$ Finally, the set-alternation effect did not enter into any interaction with the $n-1$ response-repetition variable (all $\left.F_{\mathrm{S}}<1.5\right)$. Thus, on a general level, the finding of set-alternation costs (Mayr \& Keele, 2000) can be replicated with this paradigm.

\section{Inhibition Versus Episodic Retrieval}

To examine whether the set-alternation cost can be explained by the episodic-retrieval account, switch trials were classified by two criteria, $n-2$ rule repetitions (set alternation and no set alternation) and $n-2$ response repetition (yes or no), into the four cases illustrated in Figure 1. Figure 3 shows the relevant RTs. As is evident, there was both a substantial set-alternation cost $\left[F(1,38)=35.0, M S_{\mathrm{e}}=\right.$ $3,466.1, p<.01]$ and a smaller $n-2$ response-repetition effect $\left[F(1,38)=7.69, M S_{\mathrm{e}}=33,59.0, p<.01\right]$, whereas the interaction between the two factors was not significant $\left[F(1,38)=1.3, M S_{\mathrm{e}}=3162.2, p=.26\right] .{ }^{3}$ There were no modulations of the critical pattern associated with variations of RCI and CSI (all $\left.F_{\mathrm{S}}<1.0\right)$. Error scores were 3.7\% $(S D=3.1)$ and $3.7 \%(S D=4.6)$ for $n-2$ response changes and repetitions, in the case of $n-2$ rule changes and $4.8 \%$ $(S D=3.3)$ and $3.8 \%(S D=4.2)$ for the respective $n-2$ rule-repetition conditions; the interaction was not significant $\left[F(1,38)=1.7, M S_{\mathrm{e}}=2,012.0, p=.2\right]$. There were too few trials to enter the $n-1$ response-repetition variable into this analysis. However, when we excluded immediate response repetitions from the above analysis, there was no change in the pattern of results. Thus, as these results show, the set-alternation cost was obtained even when $n-2$ rule repetitions were accompanied by exact repetitions of stimulus- response configurations. This is consistent with the claim that the set-alternation cost reflects set-specific inhibition.

\section{CONCLUSION}

Two important results concerning set-specific inhibition were obtained. The most important finding was that set-alternation costs were found even when $n-2$ rule repetitions were associated with complete $n-2$ repetitions of stimulus-response constellations. If episodic retrieval had played a role here, RT facilitation should have occurred for $n-2$ repetitions of rules and stimulus-responses. Thus, this constitutes convincing evidence that set-level inhibition as obtained in Mayr and Keele (2000) and in the present study is attributable not to set-specific episodic retrieval but to persisting inhibition of a task set. In replicating Mayr and Keele, this inhibition appears to decay with time but not to be susceptible to reduction by endogenous preparation.

This unambiguous demonstration of inhibition on the level of task set is of relevance beyond its immediate implications in the context of task-switching research. Inhibition has been proposed as an important hypothetical process in the context of various models of cognitive con- trol. However, as is evidenced by the ongoing discussion about the interpretation of negative priming effects (e.g., Kane et al., 1997; Neill, 1997), it has been difficult to establish inhibition as an empirical phenomenon on the behavioral level. The finding of set-alternation costs (Mayr, 2001; Mayr \& Keele, 2000), along with other recent results (e.g., M. C. Anderson, Bjork, \& Bjork, 2000), is at least consistent with the notion that inhibition is critical when codes need to be selected against highly active competitors. Further, in contrast to the negative-priming paradigm, potential contributions of episodic retrieval can be assessed relatively easily in the context of the set-alternation cost. This renders the set-alternation paradigm particularly useful for further investigations of the characteristics of inhibition.

A second important result is that the finding of setalternation costs could be generalized beyond the rather limited task domain used by Mayr and Keele (2000). Specifically, set-alternation costs were obtained here with a paradigm in which changes of task sets (i.e., action rules) were not associated with changes in perceptual dimensions. This allowed the inference that set-specific inhibition is not necessarily tied to the attentional modulation of perceptual dimensions. Rather, the results are compatible with the idea that inhibition affects internal control settings that guide action on a relatively abstract level.

Finally, the fact that episodic retrieval was not responsible for the present finding of set-alternation costs does not imply that bindings between task sets and lower level parameters may not play an important role under certain circumstances. On an empirical level, it is interesting to note that, just as reported by Mayr and Keele (2000), in the present results, there actually was a numerical tendency toward a set-specific $n-2$ response-repetition benefit for both RTs (Figure 3) and errors. On a theoretical level, the idea that task sets constrain lower level associative processes is an appealing one (Allport \& Wylie, 2000). After all, outside of task-switching experiments, control settings and lower level action parameters may often covary. In order to adapt to such covariations, to associate particular action specifications and higher-level control settings would be a very useful device. Direct evidence for an associative binding process that is constrained by the currently relevant task set is missing so far. However, the finding that repetition-priming effects are turned into repetition costs in the case of a task-set switch (Figure 2; see also Quinlan, 1999; Rogers \& Monsell, 1995) could be interpreted as indirect evidence for the role of higher-level control settings in constraining lower-level associative processes.

\section{REFERENCES}

Allport, D. A., Styles, E. A., \& Hsieh, S. (1994). Shifting intentional set: Exploring the dynamic control of tasks. In C. Umiltà \& M. Moscovitsch (Eds.), Attention and performance XV: Conscious and nonconscious information processing (pp. 421-452). Cambridge, MA: MIT Press. 
Allport, [D.] A., \& Wylie, G. (2000). Task-switching, stimulusresponse bindings, and negative priming. In S. Monsell \& J. Driver (Eds.), Control of cognitive processes: Attention and performance XVIII (pp. 35-70). Cambridge, MA: MIT Press.

Amos, A. (2000). A computational model of information processing in the frontal cortex and the basal ganglia. Journal of Cognitive Neuroscience, 12, 505-519.

Anderson, J. R. (1993). Rules of the mind. Hillsdale, NJ: Erlbaum.

Anderson, M. C., BJork, E. L., \& BJork, R. A. (2000). Retrieval-induced forgetting: Evidence for a recall-specific mechanism. Psychonomic Bulletin \& Review, 7, 522-550.

Corbetta, M., Miezin, F. M., Dobmeyer, S., Shulman, G. L., \& PeTERSEN, S. E. (1991). Selective and divided attention during visual discrimination of shape, color, and speed: Functional anatomy by Positron Emission Tomography. Journal of Neuroscience, 11, 2383-2404.

GoschKe, T. (2000). Intentional reconfiguration and involuntary persistence in task-set switching. In S. Monsell \& J. Driver (Eds.), Control of cognitive processes: Attention and performance XVIII (pp. 331355). Cambridge, MA: MIT Press.

Dehaene, S., Kerszeberg, M., \& Changeux, J. A. (1998). A neuronal model of global workspace in effortful cognitive tasks. Proceedings of the National Academy of Sciences, 95, 14529-14534.

Houghton, G., \& Tipper, S. P. (1994). A model of inhibitory mechanisms in selective attention. In D. Dagenbach \& T. H. Carr (Eds.), Inhibitory mechanism in attention, memory, and language (pp.53-112). San Diego: Academic Press.

Just, M. A., \& CARPenter, P. A. (1992). A capacity theory of comprehension and individual differences in working memory. Psychological Review, 99, 122-149.

Kahneman, D., \& Tversky, A. (1972). Subjective probability: A judgement of representativeness. Cognitive Psychology, 3, 430-454.

Kane, M. J., May, C. P., Hasher, L., \& Rahal, T. (1997). Dual mechanisms of negative priming. Journal of Experimental Psychology: Human Perception \& Performance, 23, 632-650.

Kimberg, D. Y., \& Farah, M. J. (1993). A unified account of cognitive impairments following frontal lobe damage: The role of working memory in complex, organized behavior. Journal of Experimental Psychology: General, 122, 411-428.

LogAN, G. (1978). Attention in character-classification tasks: Evidence for the automaticity of component stages. Journal of Experimental Psychology: General, 107, 32-63.

MAYr, U. (2001). Age differences in the selection of mental sets: The role of inhibition, stimulus ambiguity, and response-set overlap. Psychology \& Aging, 16, 96-109.

MAYr, U., \& KeEle, S. W. (2000). Changing internal constraints on action: The role of backward inhibition. Journal of Experimental Psychology: General, 129, 4-26.

MAYr, U., \& KLIEGL, R. (2000). Task-set switching and long-term memory retrieval. Journal of Experimental Psychology: Learning, Memory, \& Cognition, 26, 1124-1140.

Meiran, N. (1996). Reconfiguration of processing mode prior to task performance. Journal of Experimental Psychology: Learning, Memory, \& Cognition, 22, 1423-1442.
NeILL, W. T. (1997). Episodic retrieval in negative priming and repetition priming. Journal of Experimental Psychology: Learning, Memory, \& Cognition, 23, 1291-1305.

Quinlan, P. T. (1999). Sequential effects in auditory choice reaction time tasks. Psychonomic Bulletin \& Review, 6, 297-303.

Rogers, R. D., \& Monsell, S. (1995). Costs of a predictable switch between simple cognitive tasks. Journal of Experimental Psychology: General, 124, 207-231.

Rubinstein, J. S., Meyer, D. E., \& Evans, J. E. (2001). Executive control of cognitive processes in task switching. Journal of Experimental Psychology: Human Perception \& Performance, 27, 763-797.

Shallice, T. (1994). Multiple levels of control processes. In C. Umiltà \& M. Moscovitsch (Eds.), Attention and performance XV: Conscious and nonconscious information processing (pp.395-420). Cambridge, MA: MIT Press.

Tipper, S. P., \& CRAnston, M. (1985). Selective attention and priming: Inhibitory and facilitatory effects of ignored primes. Quarterly Journal of Experimental Psychology, 37, 591-611.

Woodworth, R. S. (1938). Experimental Psychology. New York: Holt.

\section{NOTES}

1. The reason the fully orthogonal design with independent manipulation of RCI and CSI was not chosen here is that these two factors do not allow independent manipulation of the two theoretically critical parameters-namely, passive decay time and time for preparation. The reason is that RCI affects decay time only, whereas CSI affects decay and preparation. With the three conditions implemented, theoretically meaningful tests can be conducted concerning the role of passive decay (i.e., $150-150$ vs. $650-150$ ) and the role of active preparation (i.e., $650-150$ vs. $150-650)$. Specifically, the latter contrast keeps the overall decay time constant while varying the preparation time.

2. A sequential expectancy account may explain task-set-alternation costs by assuming that participants are biased toward expecting $\mathrm{A}-\mathrm{B}-\mathrm{C}$ type of sequences over A-B-A type of sequences on the basis of a "representativeness heuristic within small numbers" (e.g., Kahneman \& Tversky, 1972). However, it is hard to see how such expectancies could be upheld in the case of a $100 \%$ valid task cue and sufficient time for preparation.

3 . In the case of $n-2$ set repetitions, $n-2$ repetitions of stimulus and response locations always coincide. In contrast, for $n-2$ set change sequences, stimulus and response repetitions are always separate events, raising the possibility that $n-2$ response repetitions are not the adequate control for the critical $n-2$ stimulus + response repetitions. However, a separate analysis revealed that the $n-2$ stimulus-repetition effect for $n-2$ set change sequences was only $4 \mathrm{msec}$ and was far from significant $\left[F(1,38)=0.4, M S_{\mathrm{e}}=778.2, p>.5\right]$.

(Manuscript received February 23, 2000; revision accepted for publication June 1, 2001.) 